\title{
Giant venous graft aneurysm identified by coronary angiography and three-dimensional computed tomography angiography
}

\author{
Michal Chmielecki ${ }^{1}$, Milosz Jaguszewski ${ }^{1}$, Aneta Strozyk ${ }^{1}$, \\ Jerzy Gorny ${ }^{2}$, Adam Kern ${ }^{2,3}$, Marcin Gruchala ${ }^{1}$, Dariusz Ciecwierz ${ }^{1}$ \\ ${ }^{1} 1^{\text {st }}$ Department of Cardiology, Medical University of Gdansk, Poland \\ ${ }^{2}$ Department of Cardiology, Voivodal Specialist Hospital in Olsztyn, Poland \\ ${ }^{3}$ Faculty of Medical Sciences, University of Warmia and Mazury, Olsztyn, Poland
}

A 60-year-old obese male patient was admitted to the coronary care unit complaining of typical angina (CCS4). The patient's history included chronic renal failure and subsequent kidney transplantation, long-term hypertension, and coronary artery disease. In 1990, he underwent surgical revascularization, where 3 venous grafts to left artery descending (LAD), circumflex artery (Cx), and right coronary artery were implanted. After 13 years the patient was referred for a complex percutaneous coronary intervention (PCI) including bare-metal stent (BMS) implantation to $\mathrm{Cx}$, marginal branch and saphenous graft to LAD. At presentation, standard electrocardiogram revealed no new abnormalities with troponin T of $0.1 \mathrm{ng} / \mathrm{mL}$. Echocardiography showed a pathological extracardiac structure adjacent to the left ventricle anterior wall (Fig. 1A, B). The subsequent angiography revealed a significant multivessel disease comprising a severe stenosis of the distal portion of $\mathrm{Cx}$ and a giant $(80 \times 50 \mathrm{~mm})$ saphenous venousgraft aneurysm (SVGA) to LAD with intramural thrombus formations, in-stent restenosis, and preserved Thrombolysis in Myocardial Infarction grade 3 flow (Fig. 1C). Three-dimentional computed tomography angiography (CTA) (Fig. 1D) and exercise-induced myocardial ischemia with Tc-99m sestamibi single-photon emission computed tomography were performed for the purpose of precise structural evaluation of the venous graft and myocardial perfusion in the area of LAD, respectively. Considering the high risk of reoperation and no substantial anterior wall ischemia the patient was offered a percutaneous treatment of $\mathrm{Cx}$. The PCI and subsequent BMS implantation was performed with an excellent final result. After the procedure, the patient remained asymptomatic and discharged home in a good clinical condition. The long-term follow-up was uneventful.

Aneurysmal dilatation of saphenous vein graft remains an extremely rare surgical complication after coronary artery by-pass graft procedure. Although no screening guideline related to the aortocoronary SVGA exists, the incidence has been ascertained to be around $0.07 \%$ in a single center experience. In several cases, the symptoms of myocardial ischemia were documented owing to a distal embolization, fistula drainage, mass effect in the native vessel, or even rupture resulting in hemodynamic compromise [1-4]. Nevertheless, SVGAs, by their asymptomatic course, seem to be underdiagnosed and are mainly identified accidentally on imaging [5]. Previously, patients with SVGAs were referred for surgical strategy. With refinement of percutaneous techniques, treatment strategies in patients with SVGAs are diverse, comprising, implantation of Amplatzer device, stentgrafts, and arterial coils [6]. Recently, several novel techniques have been described, including the transluminal reconstruction, to seal the aneurysm and restore distal flow [7-10]. Since there is no consensus on the optimal approach in patients with SVGA, Ramirez et al. [6] proposed management algorithm identifying individuals best suited for percutaneous or surgical treatment strategy [3]. There is no doubt that all these patients, if possible, should be assessed by the heart-team. The first step in deciding on management options for SVGAs is to assess for the presence of mechanical complications and/or other surgical indication. In cases with patent SVGAs and suitable anatomy, percutaneous management with a covered stent

Address for correspondence: Michal Chmielecki, $\mathrm{MD}, \mathrm{PhD}, 1^{\text {st }}$ Department of Cardiology, Medical University of Gdansk, ul. Dębinki 7, 80-952 Gdańsk, Poland, e-mail: mchmielecki@gumed.edu.pl 


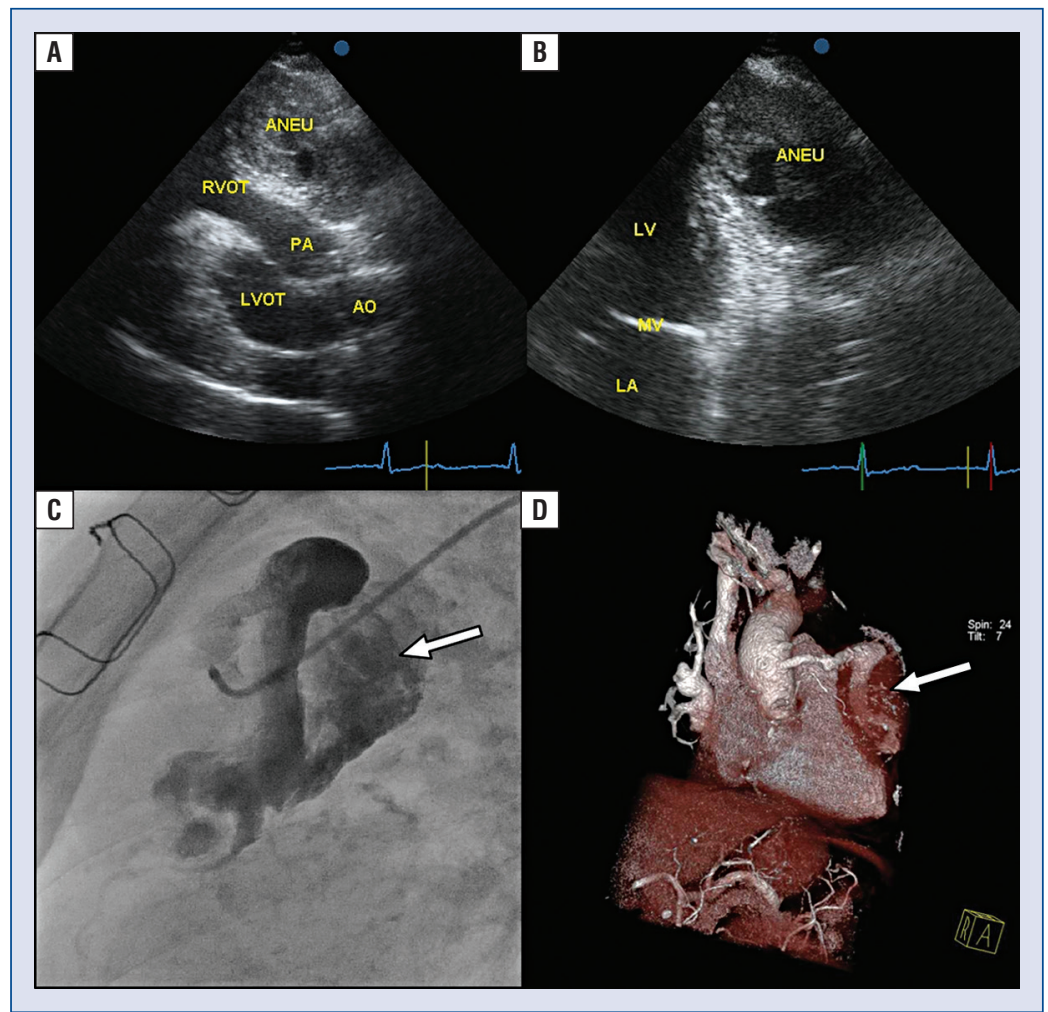

Figure 1. A giant aorta (AO)-left artery descending graft aneurysm demonstrated by transthoracic echocardiography (A, B), angiography (C, arrow) and three-dimensional computed tomography angiography (D, arrow); ANEU — aneurysm; LA — left atrium; LV - left ventricle; MV — mitral valve; LVOT - left ventricular outflow tract; RVOT — right ventricular outflow tract; PA — pulmonary artery.

should be pursued [3]. Multimodality imaging is often required to establish the size of the SVGA and the area supplied by affected graft [11]. Last but not least, the assessment of clinical symptoms and myocardial viability plays a key role in this diverse setting.

What sets our this case apart from most other ones is the fact of recording an unusual size of aneurysm, which sometimes can be hardly distinguished from pseudoaneurysm, even on the CTA scans. Any surgical intervention on a degenerated bypass was exposed to unreasonable risk of a massive intraoperative bleeding. Thus, since the imaging examination did not reveal any essential ischemia of the anterior wall, to avoid reoperation in this high-risk patient, conservative management was sought, following heart team discussion achieving a satisfactory long-tem clinical result.

\section{Conflict of interest: None declared}

\section{References}

1. Jukema JW, van Dijkman PR, van der Wall EE. Pseudoaneurysm of a saphenous vein coronary artery bypass graft with a fistula draining into the right atrium. Am Heart J, 1992; 124: 1397-1399.
2. Sugimoto T, Yamamoto K, Yoshii S et al. Large saphenous vein graft aneurysm with a fistula to the right atrium. Ann Thoracic Cardiovasc Surg, 2006; 12: 435-437.

3. Garcia-Lara J, Pinar-Bermudez E, Hurtado JA, Valdez-Chavarri M. Giant true saphenous vein graft aneurysm. J Am Coll Cardiol, 2009; 54: 1899.

4. Hudzik B, Glowacki J, Szkodzinski J, Wasilewski J, Majsnerowska A, Polonski L. Giant saphenous vein graft pseudoaneurysm compressing the right atrium and right ventricle and presenting as decompensated heart failure. Can J Cardiol, 2011; 27: 390 e9-11.

5. Dieter RS, Patel AK, Yandow D et al. Conservative vs. invasive treatment of aortocoronary saphenous vein graft aneurysms: Treatment algorithm based upon a large series. Cardiovasc Surg, 2003; 11: 507-513.

6. Ramirez FD, Hibbert B, Simard T et al. Natural history and management of aortocoronary saphenous vein graft aneurysms: A systematic review of published cases. Circulation, 2012; 126: 2248-2256.

7. Patterson T, Webb I, Redwood SR. Percutaneous luminal reconstruction of giant saphenous vein graft aneurysm. J Am Coll Cardiol Cardiovasc Interv, 2015; 8: e141-2.

8. Vizzi V, Bucciarelli-Ducci C, Johnson TW et al. Giant saphenous vein graft aneurysm: A complex multi-disciplinary percutaneous approach. Int J Cardiol, 2015; 182: 384-386.

9. Ali N, Mathew T, Henderson R, Agostoni P, Werner N. How should I treat an asymptomatic enlarging giant saphenous vein graft aneurysm? EuroIntervention, 2014; 9: 1244-1247.

10. Brooks MJ, Grigg L, Mitchell P et al. Percutaneous closure of a giant saphenous vein graft aneurysm with an Amplatzer vascular plug. J Am Coll Cardiol Cardiovasc Interv, 2013; 6: 420-422.

11. del Castillo Carnevali H, Recio Vazquez M, Moya Mur JL, Barrios Alonso V, Zamorano JL. Giant pseudoaneurysm of a saphenous vein graft. Eur Heart J Cardiovasc Imaging, 2012; 13: 1053. 